\title{
Inhaltsverzeichnis
}

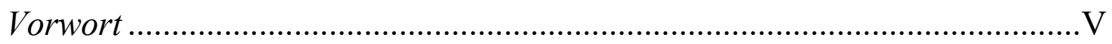

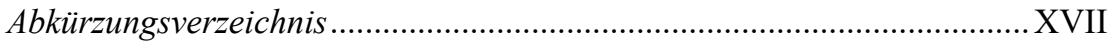

\section{ERSTER TEIL: \\ EINLEITUNG}

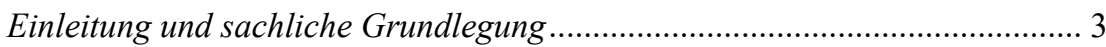

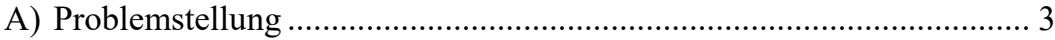

B) Methoden und Fragestellungen........................................................... 8

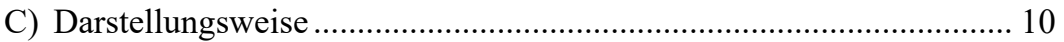

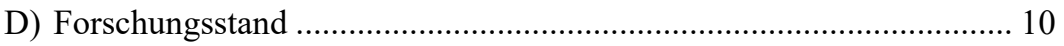

\section{ZWEITER TEIL: \\ KODIFIZIERUNG DES COMPUTERSTRAFRECHTS}

Erstes Kapitel: Technisch-phänomenologische Grundlegung ........................ 15

A) Zentrale Entwicklungsetappen der Computertechnik .......................... 15

I. Anstieg der Abhängigkeit von der Technik................................ 15

II. Einstellungswandel gegenüber der Technik ................................. 23

III. Veränderungen beim Täterkreis ................................................... 29

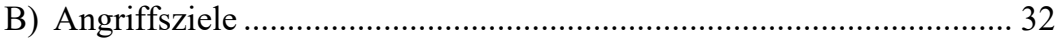

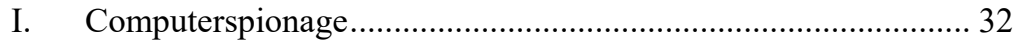

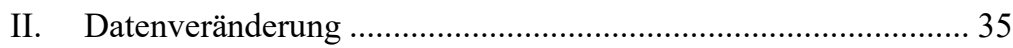

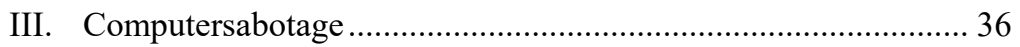

IV. Verwendung des Hacking zur Tatbegehung................................. 39

C) Charakteristika der Computerkriminalität ............................................. 43

Zweites Kapitel: Legislatorische Grundlegungdas Zweite Gesetz zur Bekämpfung der Wirtschaftskriminalität ................ 48

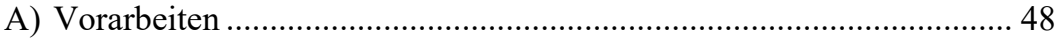

I. Erste Einschätzungsergebnisse des Bundesministeriums

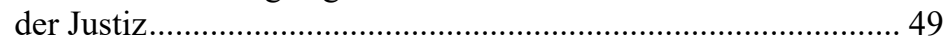

II. Die Positionierung der Sachverständigenkommission ................. 51 


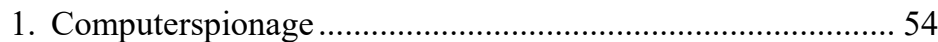

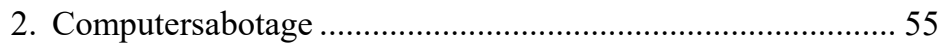

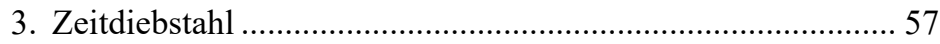

B) Gesetzgebungsverfahren zum 2. WiKG........................................... 58

I. Beratungsergebnisse des Rechtsausschusses vom 6. Juni 1984..... 60

1. Phänomenologische Besonderheiten

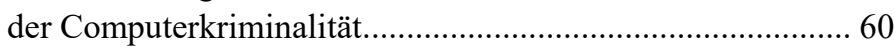

2. Entwicklungstendenzen ............................................................. 63

3. Überlegungen zu präventiven Ansätzen ................................... 67

4. Reformansätze für die Implementierung eines

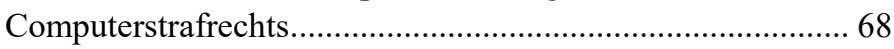

a) Computerspionage …………………………………...... 70

b) Computersabotage ........................................................ 71

c) Zeitdiebstahl..................................................................... 72

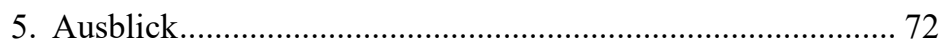

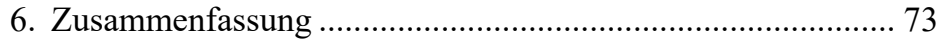

II. Diskussionsschwerpunkte der folgenden Beratungen .................. 73

1. Computerspionage ………………………………................ 74

a) Kodifizierung von Begriffsdefinitionen ............................ 75

b) Das Erfordernis der besonderen Sicherung ....................... 77

c) Die Höhe des Strafrahmens................................................ 78

d) Die Ausgestaltung als Antragsdelikt .................................. 79

e) Zwischenergebnis zur tatbestandlichen Fassung

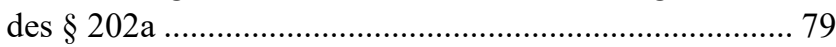

f) Positionierung zum Hacking ............................................ 79

2. Computersabotage ................................................................ 84

a) Erhöhung des Strafrahmens .............................................. 85

b) Zum Erfordernis des Tatbestandsmerkmals „,fremd“....... 86

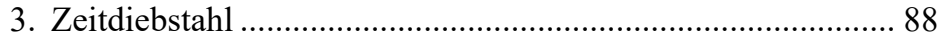

III. Beschlussempfehlung des Rechtsausschusses ............................. 88 


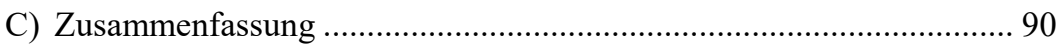

I. Inhalt und Motive für die Einführung des $\S 202$ a i.d.F.

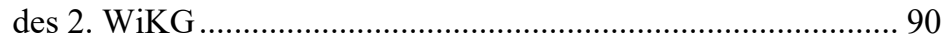

II. Inhalt und Motive für die Einführung des $\S 303$ a i.d.F.

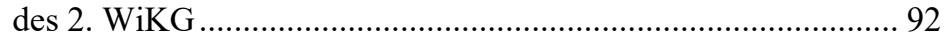

III. Inhalt und Motive für die Einführung des $\S 303 b$ i.d.F.

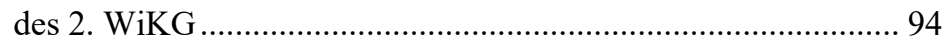

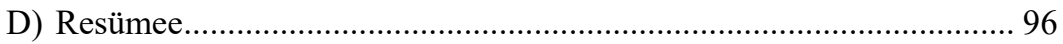

\section{DRITTER TEIL: \\ KODIFIZIERUNG DES INTERNETSTRAFRECHTS}

Drittes Kapitel: Technisch-phänomenologische Grundlegung ..................... 101

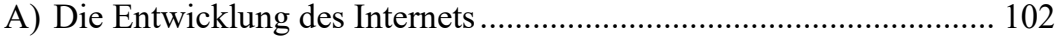

I. Wandel vom militärischen zum wissenschaftlichen Netzwerk.... 102

II. Von der passiven Kopplung zum interaktiven

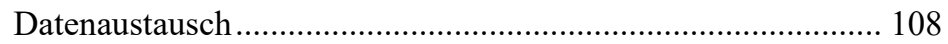

III. Übergang vom Wissensspeicher zum

Kommunikationsmedium ...................................................... 110

IV. Phase des sog. Internetting ...................................................... 111

V. Zusammenschluss zum „Netz der Netze“.................................. 116

VI. Wandel zum Massenmedium.................................................... 119

VII. Zusammenfassung ................................................................... 121

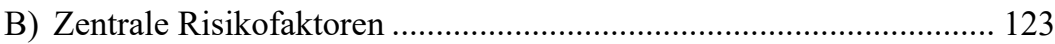

I. Datenübertragungstechnik als struktureller Risikofaktor .......... 123

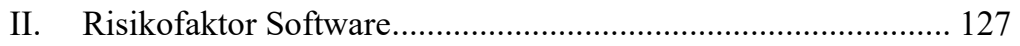

III. Anwender als personelle Risikofaktoren ..................................... 130

C) Phänomenologische Besonderheiten der Internetkriminalität............ 132

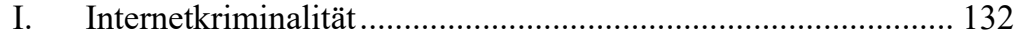

II. Gefährdete Interessen ............................................................. 133

1. Vertraulichkeitsverletzungen .............................................. 134

2. Beeinträchtigungen der Integrität und Verfügbarkeit ........... 140 


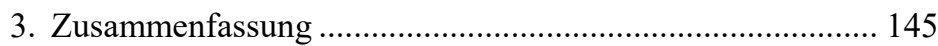

III. Empirisch-kriminologische Charakteristika ............................ 146

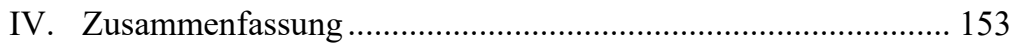

Viertes Kapitel: Initiativen des Europarates ................................................. 156

A) Die Empfehlung Nr. R (89) 9 vom 13. September 1989.................... 157

I. Die Bewertung der Ausgangssituation ..................................... 158

II. Allgemeine Ausführungen zur Ausgestaltung der Empfehlung...... 159

III. Materiell-rechtliche Vorgaben der Empfehlung ........................ 161

1. Unbefugter Zugang.............................................................. 162

2. Unberechtigtes Abfangen von Daten ..................................... 163

3. Beeinträchtigung von Computerdaten und -programmen..... 164

4. Eingriffe in Computersysteme …………………………...... 167

5. Ergänzende Ausführungen.................................................... 168

B) Die Convention on Cybercrime vom 23. November 2001.................. 168

I. Allgemeine Ausführungen zum Regelungsgegenstand der Konvention ..................................................................... 172

II. Grundlegende Begriffsbestimmungen ......................................... 173

III. Materiell-rechtliche Vorgaben zu den sog. CIA-Delikten........... 176

1. Rechtswidriger Zugang gem. Art. 2 CCC............................. 176

2. Rechtswidriges Abfangen gem. Art. 3 CCC .......................... 179

3. Eingriff in Daten gem. Art. 4 CCC ........................................ 180

4. Eingriff in ein System gem. Art. 5 CCC............................... 182

5. Missbrauch von Vorrichtungen gem. Art. 6 CCC ................ 184

6. Weitergehende Vorgaben ....................................................... 186

IV. Ausblick zum Anpassungsbedarf für das deutsche Strafrecht.... 186

V. Die Bedeutung und Tragweite der Konvention ......................... 187

Fünftes Kapitel: Initiativen der Europäischen Union ................................... 191

A) Die erste strafrechtliche Harmonisierungswelle ................................ 191

B) Die zweite Phase der Strafrechtsharmonisierung............................... 195 
I. Frühere Aktivitäten der Kommission......................................... 196

II. Die Erarbeitung des Kommissionsvorschlags ........................... 197

III. Inhalt des Kommissionsvorschlags vom 19. April 2002 ............ 198

1. Allgemeine Begriffsbestimmungen in Art. 2 des RB ........... 200

2. Materiell-rechtliche Vorgaben zu den CIA-Delikten............ 202

IV. Stellungnahmen der Ausschüsse des Europäischen Parlaments .... 205

1. Ergänzungsvorschläge der Ausschüsse ................................. 206

2. Begründungen zu den Stellungnahmen ................................. 209

V. Die Fortsetzung des Verfahrens im Rat der Europäischen Union ................................................................ 210

C) Die inhaltliche Ausgestaltung der Schlussfassung............................. 211

I. Änderungen bei den Begründungserwägungen ......................... 211

II. Änderungen zu den Begriffsbestimmungen in Art. 1 des RB ... 212

III. Anpassungen bei der Computerspionage in Art. 2 des RB........ 213

IV. Neuerungen zur Computersabotage in Art. 3 des RB ................. 214

V. Gesonderte Kodifizierung der Datenveränderung in

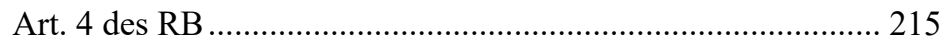

VI. Änderungen bei der Versuchsstrafbarkeit in Art. 5 des RB....... 216

VII. Vorgaben zum Strafrahmen in Art. 6 und Art. 7 des RB........... 216

VIII. Sonstige Anpassungen............................................................ 219

IX. Zusammenfassung der grundlegenden Änderungsansätze

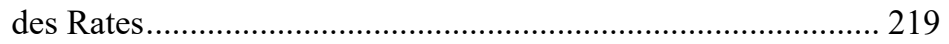

D) Die Rechtswirkung des Rahmenbeschlusses 2005/222/JI ................. 220

E) Zusammenfassende Beurteilung der Kennzeichen der zweiten Phase der Strafrechtsharmonisierung ................................... 224

F) Die dritte Phase der Strafrechtsharmonisierung................................. 225

I. Strukturell bedingte Interessenlage der klagenden Kommission.....226

II. Inhalt des EuGH-Urteils in der Rs. C-176/03 .............................. 227

III. Zusammenfassung zur Rs. C-176/03 ........................................ 230

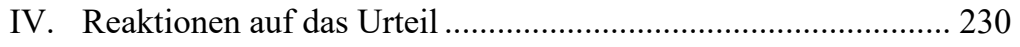


1. Mitteilung der Kommission vom 23. November 2005 230

2. Die Bewertung durch das Europäische Parlament

3. Die Stellungnahme des Bundesrates vom 10. Februar 2006

4. Reaktion des EuGH im Urteil zur Rs. C-440/05

V. Bedeutung der EuGH-Rechtsprechung für die dritte Welle der Strafrechtsharmonisierung.

G) Gemeinsamkeiten und Unterschiede zwischen der Convention on Cybercrime und dem Rahmenbeschluss

I. Mindestvorgaben hinsichtlich der Grundtermini gem. Art. 1 CCC und Art. 1 des RB

II. Computerspionage gem. Art. $2 \mathrm{CCC}$ und Art. 2 des RB 239

III. Datenveränderung gem. Art. $4 \mathrm{CCC}$ und Art. 4 des RB. 240

IV. Computersabotage gem. Art. 5 CCC und Art. 3 des RB 240

V. Versuch, Beihilfe und Anstiftung gem. Art. 11 CCC und Art. 5 des RB

VI. Sanktionen gem. Art. 13 CCC und Art. 6 und 7 des RB

Sechstes Kapitel: Bewertung der bisherigen Strafrechtsharmonisierung ..... 242

A) Institutionelle Weiterentwicklung.... 243

B) Fortentwicklung der verwendeten Rechtsakte 244

C) Notwendigkeit einer europäischen Strafrechtsharmonisierung 245

D) Die thematische Begrenzung der Strafrechtsangleichung. 249

E) Verfahrensbezogene Kritik zum Erlass konkreter Rechtsakte 254

F) Kritik an der inhaltlichen Ausgestaltung der erlassenen Rechtsakte 257

Siebentes Kapitel: Die Umsetzung der europa- und völkerrechtlichen Vorgaben in nationales Recht

A) Das 41. Strafrechtsänderungsgesetz zur Bekämpfung der Computerkriminalität vom 7. August 2007 263

I. Inhalt des 41. Strafrechtsänderungsgesetzes................................ 265

1. Bezugnahme auf die europäischen Begriffsdefinitionen ...... 265

2. Anpassungen im Bereich der Computerspionage 266 
3. Hauptkritikpunkte in der parlamentarischen Diskussion ...... 270

4. Kodifizierung des $\S 202 b$ n.F. über das Abfangen von Daten 274

5. Geäußerte Kritik zur Neuaufnahme des $\S 202 b$ 276

6. Die Pönalisierung von Vorbereitungshandlungen

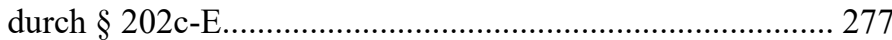

7. Die parlamentarische Debatte zu $\S 202 c-E$............................ 279

a) Kritik zur Reichweite des $\S 202 c$ Abs. 1 Nr. 2-E........... 280

b) Kritische Äußerungen zur Fallgruppe des sog. Phishing.....287

c) Stellungnahme des Rechtsausschusses 290

d) Änderungsantrag einzelner Abgeordneter und der Fraktion der Linken vom 23. Mai 2007 .................... 291

e) Zusammenfassung zur Reichweite des Art. 202c-E....... 292

8. Erste Reaktionen zur Reichweite des § 202c in der Strafrechtspraxis

a) Handhabung durch die Staatsanwaltschaft 293

b) Nichtannahmebeschluss des Bundesverfassungsgerichts vom 18. Mai 2009

c) Stellungnahmen in der Literatur.......................................... 296

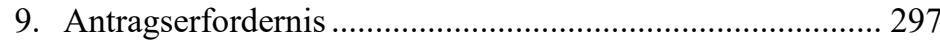

10. Vorgaben zur Datenveränderung und Computersabotage ... 298

11. Kritische Stellungnahmen zur Novellierung durch $\S \S 303 a-E$ und 303b-E ................................................. 302

a) Einwände gegen die Anpassung des § 303a a.F............. 302

b) Kritik an der Ausgestaltung des $\S 303 b-E$........................ 303

c) Änderungsantrag einzelner Abgeordneter und der Fraktion der Linken zu § 303b-E .............................. 305

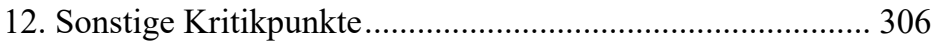

II. Die Beurteilung durch die Kommission ..................................... 307

1. Grundlegende Kritikpunkte ……………………………...... 308

2. Begrüßende Ausführungen im Schlussbericht...................... 309 
B) Das Vertragsgesetz zur Convention on Cybercrime

C) Zusammenfassung zur Umsetzung

Achtes Kapitel: Die Richtlinie 2013/40/EU vom 12. August 2013

A) Die vierte Harmonisierungswelle nach dem Vertrag von Lissabon....... 315

B) Die Richtlinie 2013/40/EU vom 12. August 2013 ............................. 318

I. Phänomenologische Veränderungen ……………………….... 318

II. Zentrale Anknüpfungspunkte des Reformvorhabens ................ 322

III. Einleitung des Gesetzgebungsverfahrens durch

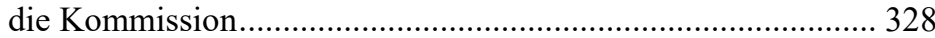

IV. Überarbeitung des Kommissionsvorschlags im Rat ................... 329

V. Zentrale Gestaltungsvorschläge................................................. 332

VI. Die Erarbeitung der Schlussfassung durch das Parlament......... 336

1. Änderungsvorschläge zu den vorangestellten Erwägungen .... 338

2. Überarbeitung der materiell-rechtlichen Vorgaben ............... 345

VII. Inhalt der Richtlinie - Gemeinsamkeiten und Unterschiede zu den bisherigen Harmonisierungsvorschriften ........................ 351

1. Gegenstand und Erwägungen für den Erlass der Richtlinie.... 351

2. Begriffsbestimmungen gem. Art. 2 der RL ........................... 353

3. Computerspionage gem. Art. 3 der RL .................................. 354

4. Computersabotage und Datenveränderung gem. Art. 4 und 5 der RL

5. Neuaufnahme des rechtwidrigen Abfangens

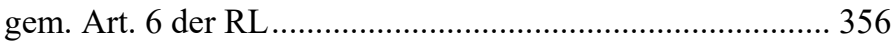

6. Aufnahme der Vorfeldstrafbarkeit in Art. 7 der RL ............. 356

7. Vorgaben zu Anstiftung, Beihilfe und Versuch

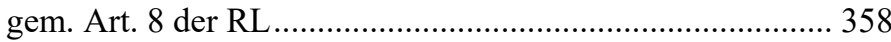

8. Anhebung des Strafrahmens gem. Art. 9 der RL ................... 358

9. Formelle Vorgaben in Art. 15 bis 19 der RL ......................... 360

C) Zusammenfassung 
Neuntes Kapitel: Umsetzung der Richtlinienvorgaben

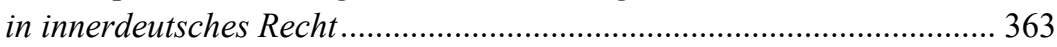

A) Strafbarkeit der Datenhehlerei gem. § 202d StGB........................... 363

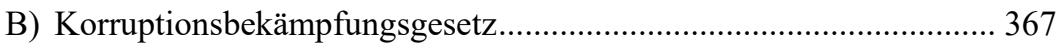

C) Gesetzentwurf zum „digitalen Hausfriedensbruch“............................ 372

I. Anknüpfungspunkt und Zielstellung des Entwurfs ................... 373

II. Schutzlücken de lege lata ......................................................... 375

III. Schutzlücken bei der Strafverfolgung ....................................... 380

IV. Stellungnahmen zum Strafrechtsschutz de lege ferenda............. 382

1. Grundtatbestand, § 202e Abs. 1 StGB-E .............................. 382

a) Zugang, § 202e Abs. 1 S. 1 Nr. 1 StGB-E ...................... 382

b) Gebrauchsrecht, § 202e Abs. 1 S. 1 Nr. 2 StGB-E......... 385

c) Beeinflussen oder Ingangsetzen, $\S 202 \mathrm{e}$ Abs. 1 S. 1 Nr. 3 StGB-E.................................... 386

2. Erhöhte Strafandrohung …………………………………....... 387

3. Versuchsstrafbarkeit und prozessuale Einkleidung ............... 390

V. Stellungnahmen zur Schutzrichtung ........................................... 390

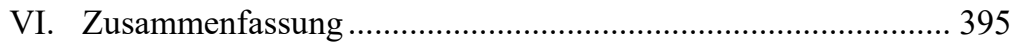

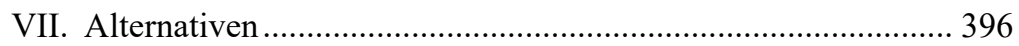

\section{VIERTER TEIL}

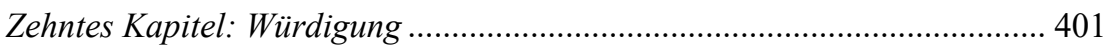

A) Technisch-phänomenologische Grundlegung .................................... 401

B) Legislatorische Grundlegung - Kodifizierung des

Computerstrafrechts durch das 2. WiKG .......................................... 404

C) Zusammenhang zwischen der technischen Weiterentwicklung und der Verlagerung gesetzgeberischer Initiativen in den

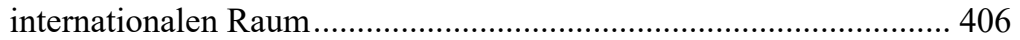

D) Völker- und europarechtliche Angleichungsbemühungen .................. 407

I. Convention on Cybercrime des Europarates vom 23. November 2001 408 
II. Der Rahmenbeschluss 2005/222/JI des Rates vom 24. Februar 2005 ............................................................. 410

III. Die Umsetzung in nationales Strafrecht .................................. 412

IV. Richtlinie 2013/40/EU vom 12. August 2013

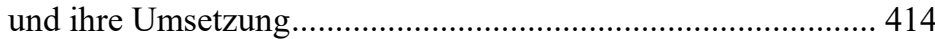

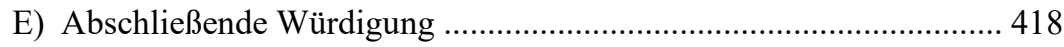

\section{ANHANG}

Quellenverzeichnis....... 423

Literaturverzeichnis 462 